\title{
Energy Transduction of Isothermal Ratchets: Generic Aspects and Specific Examples Close to and Far from Equilibrium
}

\author{
Andrea Parmeggiani, Frank Jülicher, Armand Ajdari* and Jacques Prost \\ Institut Curie, Physico-Chimie Curie, UMR CNRS/IC 168, 26 rue d'Ulm, 75248 Paris Cedex 05, France \\ * Laboratoire de Physico Chimie Théorique, Esa CNRS 7083, ESPCI, 10 rue Vauquelin, 75231 Paris Cedex 05, France
}

\begin{abstract}
We study the energetics of isothermal ratchets which are driven by a chemical reaction between two states and operate in contact with a single heat bath of constant temperature. We discuss generic aspects of energy transduction such as Onsager relations in the linear response regime as well as the efficiency and dissipation close to and far from equilibrium. In the linear response regime where the system operates reversibly the efficiency is in general nonzero. Studying the properties for specific examples of energy landscapes and transitions, we observe in the linear response regime that the efficiency can have a maximum as a function of temperature. Far from equilibrium in the fully irreversible regime, we find a maximum of the efficiency with values larger than in the linear regime for an optimal choice of the chemical driving force. We show that corresponding efficiencies can be of the order of $50 \%$. A simple analytic argument allows us to estimate the efficiency in this irreversible regime for small external forces.
\end{abstract}

PACS Numbers: 87.10.+e, 05.40.-a

(26th July 2021)

\section{INTRODUCTION}

Biological systems provide an important motivation to study the physics of active processes which on a molecular scale are able to transduce chemical energy into mechanical work and motion. Important examples are motor proteins and enzymes which move actively along DNA [1]. The properties of such systems differ in several respects from macroscopic machines and heat engines: (i) active phenomena occur on a molecular scale in a very viscous environment with overdamped dynamics, motion is thus stochastic and obeys only on average the first and second laws of thermodynamics; (ii) these systems are isothermal and operate strictly at constant temperature as they are in intimate contact with a thermal bath. In recent years, a number of theoretical approaches to describe this class of systems have been developed [2 \& 8 ].

In order to discuss the energy transduction of such systems, the concepts which have been developed for macroscopic motors have to be applied with some care. Recently, there has been a growing interest in the energetics of Brownian motors 9 211. It is the aim of this article to discuss generic aspects of energy transduction of Brownian motors driven by a chemical reaction and to provide several specific examples which reveal new and interesting properties.

The two-state models which we use [8] represent a useful paradigm for the description of energy transduction of isothermal motors in the overdamped regime. They are motivated by cytoskeletal motor proteins which move along polar and periodic filaments. Coupling a two state model to a chemical reaction, which induces transitions between the two states of the motor, leads to motion and force-generation if the chemical potential difference $\Delta \mu$ between the fuel and its reaction products is nonzero and if the system has a polar symmetry. Assuming that the chemical reservoirs coupled to a single motor are macroscopic in size, this chemical potential difference can be defined even under out-of equilibrium conditions since in this limit the reaction driving the microscopic motor affects the reservoir only weakly. Using $\Delta \mu$ as the relevant control parameter, the consumed chemical free energy by the active process is well defined. This leads to a simple definition of efficiency $\eta$ as the ratio of the mechanical work performed and the consumed chemical free energy.

We find three important results:

- The efficiency calculated for these models can be maximized far from equilibrium.

- Close to thermal equilibrium there exists a linear response regime which is important because of its universal features. We demonstrate that the dependence of the efficiency in this regime on temperature is strongly model dependent and can be non-monotonous in which case thermal fluctuations are essential for an efficient energy transduction.

- The efficiencies vanish at stalling conditions (zero average velocity) except in a singular limit where they reach the ideal value $\eta=1$.

The outline of our paper is as follows. In section II, we discuss generic aspects which are completely independent of the model chosen. We define the efficiency and identify the generalized currents and forces which allow us to write a linear response theory. We discuss the generic features of efficiency in this regime, in particular the maximal efficiency under reversible conditions and the efficiency at stalling conditions. In Section III we choose an explicit realization of the transport equations where the motor is described as a two-state model 
which is coupled to a chemical reaction and we identify the energy fluxes in the system. Section IV discusses the energy transduction properties for specifically chosen examples. We show that efficiency is typically optimized in the irreversible regime and give examples for the temperature dependence of $\eta$ when the system operates in the linear response regime. In our concluding remarks, we relate our results to biological motors and discuss alternative definitions of efficiency which have been used in the literature.

\section{ISOTHERMAL RATCHETS: GENERIC ASPECTS}

\section{A. Force, velocity and efficiency}

Motivated by linear biological motor proteins which move along a linear filament, we will consider chemically driven systems which can induce motion along a onedimensional track. The energy source is the difference of the chemical potentials $\Delta \mu$ of fuel and products. Being motivated by biological motors, we use the hydrolysis $\mathrm{ATP} \rightleftharpoons \mathrm{ADP}+\mathrm{P}$ as example $[1]$. We define

$$
\Delta \mu=\mu_{A}-\mu_{P}
$$

where $\mu_{A}$ and $\mu_{P}$ are the chemical potentials of ATP and $\mathrm{ADP}+\mathrm{P}$, respectively. In order to perform useful mechanical work, the system has to move against an external force $f_{\text {ext }}$ applied parallel to the track. In addition to the two generalized forces $\Delta \mu$ and $f_{\text {ext }}$ acting on the system, we can define two generalized velocities: (i) the average velocity of motion $v$ of the motor along the track; and (ii) the chemical reaction rate $r$ defining the average number of ATP molecules consumed per unit time. The motor can thus be characterized by the equations of state

$$
\begin{aligned}
& v=v\left(f_{\text {ext }}, \Delta \mu\right) \\
& r=r\left(f_{\text {ext }}, \Delta \mu\right)
\end{aligned}
$$

which describe the velocities of the system as a function of the generalized forces 22]. The mechanical work performed per unit time against the external force is given by

$$
\dot{W}=f_{\text {ext }} v
$$

The amount of chemical energy consumed per unit time is

$$
\dot{Q}=r \Delta \mu
$$

For a system which performs mechanical work, i.e. $f_{\text {ext }} v<0$, we can define the (mechanical) energy transduction efficiency as 23.

$$
\eta=-\frac{f_{\mathrm{ext}} v}{r \Delta \mu}
$$

Because of energy conservation, the amount of energy dissipated per unit time therefore reads:

$$
\Pi \equiv f_{\mathrm{ext}} v+r \Delta \mu
$$

From the second law of thermodynamics it follows that $\Pi$ must always be positive.

\section{B. Linear response theory}

Close to thermal equilibrium, i.e. for small forces $f_{\text {ext }} \ll T / l$ and $\Delta \mu \ll T$, where $l$ is a typical length scale of the motor and $T$ is the temperature measured in units of $k_{B}$, we can expand Eq. (3) to linear order:

$$
\begin{aligned}
& v=\lambda_{11} f_{\text {ext }}+\lambda_{12} \Delta \mu \\
& r=\lambda_{21} f_{\text {ext }}+\lambda_{22} \Delta \mu .
\end{aligned}
$$

The matrix $\lambda_{i j}$ of linear response coefficients has the following physical meaning: $\lambda_{11}$ is a mobility giving the response of the velocity to the applied force. $\lambda_{22}$ plays a similar role for fuel consumption. It describes the 'chemical admittance' or the response of the chemical reaction rate $r$ to the chemical force $\Delta \mu$. The coefficients $\lambda_{12}$ and $\lambda_{21}$ are mechano-chemical coupling coefficients which are responsible for energy transduction.

Looking at the symmetry of the problem, we find that $v$ and $f_{\text {ext }}$ transform like vectors for $x \rightarrow-x$ while $r$ and $\Delta \mu$ are scalars which do not change under inversions. As a consequence, the coefficients $\lambda_{11}$ and $\lambda_{22}$ transform as scalars while $\lambda_{12}$ and $\lambda_{21}$ are vector coefficients. The latter can be nonzero only if the system has a polar symmetry. Thus, the polarity of the system (polar filaments) is essential for motion to exist.

Calculating the dissipation rate $\Pi$ in the linear regime, we find that $\Pi$ is positive exactly if the diagonal elements are positive, $\lambda_{i i}>0$ and if the determinant is positive

$$
\lambda_{11} \lambda_{22}-\lambda_{12} \lambda_{21}>0
$$

On general grounds, we expect a symmetry relation between the Onsager coefficients if microscopic reversibility is obeyed:

$$
\lambda_{12}=\lambda_{21}
$$

This is a general result of non-equilibrium thermodynamics.

\section{Modes of operation}

Different modes of operation of the motor can be distinguished by looking at the input and output of energy of the system. The dissipation rate $\Pi$ corresponds to the total flux of energy to the thermal bath at temperature $T$. Passive regimes of the motor are those cases where 
both $r \Delta \mu$ and $f_{\text {ext }} v$ are positive: Work performed on the system is dissipated and lost.

More interesting are the active regimes where the motor transforms chemical energy into mechanical work or vice versa while dissipating only a part of the energy input. Four such active regimes exist, see Fig. 1:

A: $r \Delta \mu>0, f_{\text {ext }} v<0$, The motor uses the chemical energy of the ATP in excess as input and performs mechanical work moving with $v>0$ against a negative force $f_{\text {ext }}<0$.

B: $r \Delta \mu<0, f_{\text {ext }} v>0$, The motor produces ATP, although already in excess, from mechanical input due to a negative force $f_{\text {ext }}<0$ inducing a negative velocity $v<0$.

C: $r \Delta \mu>0, f_{\text {ext }} v<0$, The motor uses ADP in excess to perform mechanical work.

D: $r \Delta \mu<0, f_{\text {ext }} v>0$, The motor produces ADP already in excess from mechanical work.

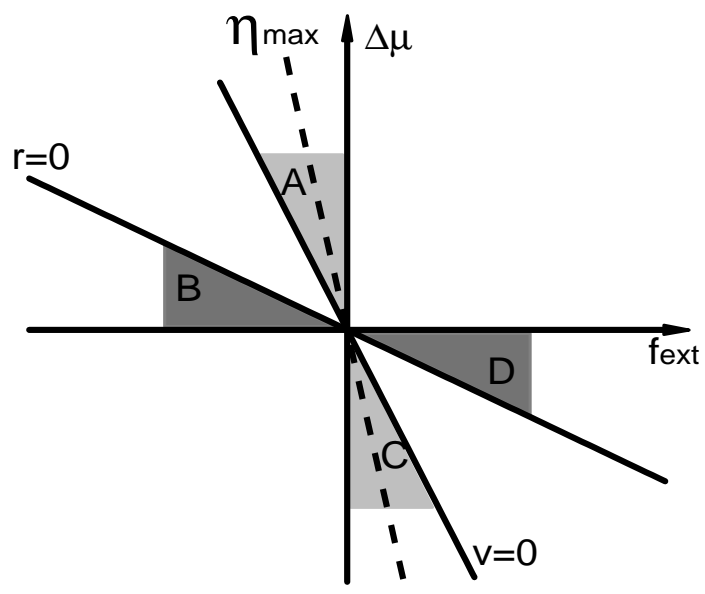

Figure 1. Operation diagram for an isothermal motor in the linear response regime as a function of external force $f_{\text {ext }}$ and chemical potential difference $\Delta \mu$. General case with four different regimes A-D, separated by lines $v=0$ and $r=0$ where the velocity and the fuel consumption vanish, respectively. The maximal efficiency occurs along a line $\eta_{\max }$.

The different regimes are separated by the lines $f_{\text {ext }}=$ $0, \Delta \mu=0, v=0$ and $r=0$. For regimes $\mathrm{A}$ and $\mathrm{C}$, where the motor performs mechanical work the mechanical efficiency is the one defined in Eq. (6): $\eta=-f_{\text {ext }} v / r \Delta \mu$. Similarly, in regimes B and D, where the system performs chemical work, the chemical efficiency $\eta_{c} \equiv-r \Delta \mu / f_{\text {ext }} v$ is more useful.

Within the linear response regime, the efficiency can be calculated using the Onsager coefficients

$$
\eta=-\frac{\lambda_{11} a^{2}+\lambda_{12} a}{\lambda_{21} a+\lambda_{22}}
$$

where $a=f_{\text {ext }} / \Delta \mu$. If we choose a constant $\Delta \mu>0$, the efficiency vanishes for $f_{\text {ext }}=0$ (no work is performed). $\eta$ becomes positive for $f_{\text {ext }}<0$ (note the minus sign which indicates that the force is applied in the direction opposing movement), reaches a maximum for a certain value of the force and becomes zero again at the stall force for which $v=0$. According to Eq. (11), the efficiency is constant along straight lines $f_{\text {ext }}=a \Delta \mu$ which correspond to constant $a$. Thus, at the origin of the $\left(f_{\text {ext }}, \Delta \mu\right)$-plane which corresponds to thermal equilibrium and reversible, quasistatic operation, the efficiency $\eta$ has a singularity and is multi-valued.

Maximal efficiency occurs for a certain value $a$ for which $\partial \eta / \partial a=0$. It is given by 24

$$
\eta_{\max }=(1-\sqrt{1-\Lambda})^{2} / \Lambda
$$

Here, $\Lambda \equiv \lambda_{12}^{2} /\left(\lambda_{11} \lambda_{22}\right)$. It varies between $\eta_{\max }=0$ for $\lambda_{12}=0$ and $\eta_{\max }=1$ if $\lambda_{12}^{2}=\lambda_{11} \lambda_{22}$. Larger values $\Lambda>1$ violate thermodynamics according to Eq. (9) and the Onsager relation (see Eq. (10)).

These arguments demonstrate that the efficiency vanishes under stalling conditions $v=0$. This is an important difference from Carnot engine for which the efficiency is optimized under quasistatic conditions without net motion. It results from the fact that the energy transduction driven by a chemical reaction considered here will in general still have a nonzero consumption rate $r$ even when motion stops, or in other words $v=0$ and $r=0$ do not occur for the same conditions.

There is however one limiting case where this is no longer true: If $\Lambda \rightarrow 1$, the two lines $r=0$ and $v=0$ in the $\left(f_{\text {ext }}, \Delta \mu\right)$-plane tend towards each other. In this limit the chemical reaction and motion are strictly coupled (i.e. one can't occur without the other) and the efficiency reaches the maximum $\eta=1$. This situation is an idealized case which applies to good approximation to polymerization forces and motion generated by polymerization processes as in the case of RNA polymerase 25, 15

\section{TWO STATE MODEL}

\section{A. Transport equations}

We study energy transduction and efficiencies of isothermal motors using simple two-state models. The motor is characterized by its position $x$ along a one dimensional coordinate describing the polar and periodic track. We assume that the motor exists in two different conformations or states $\sigma=1,2$. The interaction between motor and track depends on $\sigma$ and is described by potentials $W_{\sigma}(x)$ with polar symmetry which are periodic with period $l$.

The role of the chemical reaction is to trigger transitions between the two states. We introduce the position 
dependent rate constants $\omega_{1}(x)$ and $\omega_{2}(x)$ which characterize the probability per unit time for the transitions $1 \rightarrow 2$ and $2 \rightarrow 1$ at position $x$, respectively. The probability densities $P_{1}(x, t)$ and $P_{2}(x, t)$ for the system to be at time $t$ at position $x$ in one of the two states obey the Fokker-Planck Equations [6]

$$
\begin{aligned}
& \partial_{t} P_{1}+\partial_{x} J_{1}=-\omega_{1}(x) P_{1}+\omega_{2}(x) P_{2} \\
& \partial_{t} P_{2}+\partial_{x} J_{2}=\omega_{1}(x) P_{1}-\omega_{2}(x) P_{2}
\end{aligned}
$$

The particle currents are given by

$$
J_{\sigma} \equiv \xi^{-1}\left[-T \partial_{x} P_{\sigma}-P_{\sigma} \partial_{x} W_{\sigma}+P_{\sigma} f_{\text {ext }}\right],
$$

where $\xi^{-1}$ is an effective mobility, the temperature $T$ is measured in units of $k_{B}$ and $f_{\text {ext }}$ is the external force introduced above.

For given rates $\omega_{\sigma}$ the system relaxes to a steady state with $\partial_{t} P_{\sigma}=0$. The normalized distributions which satisfy periodic boundary conditions $\left(\int_{0}^{l} d x\left(P_{1}+P_{2}\right)=1\right.$, $P_{\sigma}(0)=P_{\sigma}(l)$ and $\left.\partial_{x} P_{\sigma}(0)=\partial_{x} P_{\sigma}(l)\right)$ in the steady state allow us to calculate the average velocity

$$
v=\int_{0}^{l} d x\left(J_{1}+J_{2}\right)
$$

\section{B. Coupling to a chemical reaction}

We now consider the situation where the transitions between states 1 and 2 occur as a result of a chemical reaction scheme which we model separately. In order to be general and to capture different situations, we consider the following scheme:

$$
\begin{aligned}
& A T P+M_{1} \underset{\alpha_{2}}{\stackrel{\alpha_{1}}{\rightleftharpoons}} M_{2}+A D P+P \\
& A D P+P+M_{1} \underset{\gamma_{2}}{\stackrel{\gamma_{1}}{\rightleftharpoons}} M_{2}+A T P \\
& M_{1} \underset{\beta_{2}}{\stackrel{\beta_{1}}{\rightleftharpoons}} M_{2}
\end{aligned}
$$

where $\alpha_{i}, \gamma_{i}$ and $\beta_{i}$ denote the forward and backward rates, respectively. The reaction pathway $\alpha$ involves ATP hydrolysis with chemical free energy gain $\Delta \mu$ when changing from state 1 to state 2 , while pathway $\gamma$ involves hydrolysis in the opposite direction. The transitions $\beta$ are do not involve a chemical potential difference. Chemical kinetics requires

$$
\begin{aligned}
& \frac{\alpha_{1}}{\alpha_{2}}=e^{\left(W_{1}-W_{2}+\Delta \mu\right) / T} \\
& \frac{\gamma_{1}}{\gamma_{2}}=e^{\left(W_{1}-W_{2}-\Delta \mu\right) / T} \\
& \frac{\beta_{1}}{\beta_{2}}=e^{\left(W_{1}-W_{2}\right) / T} .
\end{aligned}
$$

The transition rates can therefore be written as

$\omega_{1}=\alpha_{2} e^{\left(W_{1}-W_{2}+\Delta \mu\right) / T}+\gamma_{2} e^{\left(W_{1}-W_{2}-\Delta \mu\right) / T}+\beta_{2} e^{\left(W_{1}-W_{2}\right) / T}$

$\omega_{2}=\alpha_{2}+\gamma_{2}+\beta_{2}$,

where unknown (l-periodic) functions $\alpha_{2}(x), \gamma_{2}(x)$ and $\beta_{2}(x)$ define the conformation dependence of transitions rates [26]. With these expressions, the net steady state ATP consumption rate is given by

$r=\int_{0}^{l} d x\left[\left(\alpha_{1}(x)-\gamma_{1}(x)\right) P_{1}(x)-\left(\alpha_{2}(x)-\gamma_{2}(x)\right) P_{2}(x)\right]$.

\section{Detailed balance}

If $\Delta \mu=0$, the chemical reaction is in equilibrium and the transition rates are just thermal fluctuations and obey the relation of detailed balance $\omega_{1} / \omega_{2}=\exp \left(\left(W_{1}-\right.\right.$ $\left.\left.W_{2}\right) / T\right)$. Breaking of detailed balance for $\Delta \mu \neq 0$ is a requirement for spontaneous motion and force generation to be possible. In order to quantify the departure from thermal equilibrium and the extend to which detailed balance is broken, we define the quantity

$$
\Omega(x)=\omega_{1}(x)-\omega_{2}(x) \exp \left(-\frac{\Delta W(x)}{T}\right),
$$

with $\Delta W(x)=W_{2}(x)-W_{1}(x)$. Detailed balance is obeyed only if $\Omega(x)=0$ for all $x$. Using the transition rates as given by Eq. (22), we find

$\Omega(x)=e^{-\Delta W / T}\left[\alpha_{2}(x)\left(e^{\Delta \mu / T}-1\right)+\gamma_{2}(x)\left(e^{-\Delta \mu / T}-1\right)\right]$.

If $\Delta \mu \neq 0$, we distinguish two interesting limits: for small $\Delta \mu / T \ll 1$

$$
\Omega(x) \simeq\left(\alpha_{2}(x)-\gamma_{2}(x)\right) e^{-\Delta W / T} \frac{\Delta \mu}{T},
$$

indicating that $\Omega$ is proportional to $\Delta \mu$. If $\Delta \mu / T$ is large compared to one, $\Omega$ depends only on the ratio $k=[A T P] /[A D P][P]:$

$$
\Omega(x) \simeq\left(\alpha_{2}(x) e^{\Delta \mu^{0} / T} k+\gamma_{2}(x) e^{-\Delta \mu^{0} / T} k^{-1}\right),
$$

where $\Delta \mu^{0}=\mu_{A T P}^{0}-\mu_{A D P}^{0}-\mu_{P}^{0}$. Here, we used the relation $\mu_{i}=\mu_{i}^{0}+T \ln [i]$ where $[i]$ is the concentration of species $i$, and $\mu_{i}^{0}$ the so called standard chemical potential. 


\section{Energy conservation and dissipation}

The first law of thermodynamics requires that the energy flow through the system is conserved as described by Eq. (7). This energy conservation can be derived from the transport equations. This leads to expressions for the local density of energy dissipation which gives interesting insights in how energy transduction is occurring.

We distinguish two types of dissipation rates: (i) the dissipation rates $\Pi_{\sigma}$, with $\sigma=1,2$ corresponding to sliding within the potential profiles and (ii) the dissipation rates $\Pi_{\mu}$, with $\mu=\alpha, \beta, \gamma$ corresponding to transitions between the two states. In addition to the total dissipation rates $\Pi_{\sigma}$ and $\Pi_{\mu}$, we introduce local dissipation densities $\Theta_{\sigma}(x)$ and $\Theta_{\mu}(x)$ with $\Pi=\int_{0}^{l} d x \Theta(x)$.

For a particle sliding in the potential $W_{\sigma}(x)$ with a steady state distribution $P_{\sigma}(x)$ 27,28]

$$
\Theta_{\sigma}=-J_{\sigma} \partial_{x} H_{\sigma}
$$

and

$$
\Pi_{\sigma}=-\int_{0}^{l} d x J_{\sigma}(x) \partial_{x} H_{\sigma}(x)
$$

where

$$
H_{\sigma}(x) \equiv W_{\sigma}(x)-f_{\mathrm{ext}} x+T \ln \left(P_{\sigma}(x)\right)
$$

is an enthalpy whose gradient induces the Fokker-Planck current $J_{\sigma}$ :

$$
J_{\sigma}=-\xi^{-1} P_{\sigma} \partial_{x} H_{\sigma}
$$

Therefore, $\Pi_{\sigma}$ is positive definite as expected for a dissipation rate. Similarly, the dissipation densities corresponding to chemical transitions are given by

$$
\begin{aligned}
& \Theta_{\alpha}=\left(\alpha_{1} P_{1}-\alpha_{2} P_{2}\right)\left(H_{1}-H_{2}+\Delta \mu\right) \\
& \Theta_{\gamma}=\left(\gamma_{1} P_{1}-\gamma_{2} P_{2}\right)\left(H_{1}-H_{2}-\Delta \mu\right) \\
& \Theta_{\beta}=\left(\beta_{1} P_{1}-\beta_{2} P_{2}\right)\left(H_{1}-H_{2}\right)
\end{aligned}
$$

and

$$
\Pi_{\mu}=\int_{0}^{l} d x \Theta_{\mu}(x),
$$

for $\mu=\alpha, \beta, \gamma$. Again, $\Pi_{\alpha}, \Pi_{\beta}$ and $\Pi_{\gamma}$ are positive definite as required. For a steady state with periodic boundary conditions, we can partially integrate Eq. (29) and find together with Eq. (13)

$$
\begin{aligned}
\Pi_{1}+\Pi_{2} & =\int_{0}^{l} d x\left(H_{1} \partial_{x} J_{1}+H_{2} \partial_{x} J_{2}\right)+f_{\text {ext }} v \\
& =\int_{0}^{l} d x\left(H_{1}-H_{2}\right)\left(\omega_{1} P_{1}-\omega_{2} P_{2}\right)+f_{\text {ext }} v .
\end{aligned}
$$

Using Eqns. (14), (22) and (23), we find that, the total dissipation rate

$$
\Pi=\Pi_{1}+\Pi_{2}+\Pi_{\alpha}+\Pi_{\beta}+\Pi_{\gamma}
$$

satisfies Eq. (7) and energy conservation is obeyed.

For small $\Delta \mu$ and small $f_{\text {ext }}$, the two state model has a linear response regime which obeys the general properties required by thermodynamics. In particular it can be demonstrated that the model satisfies the symmetry relation of Eq. (10) as we describe in appendix A.
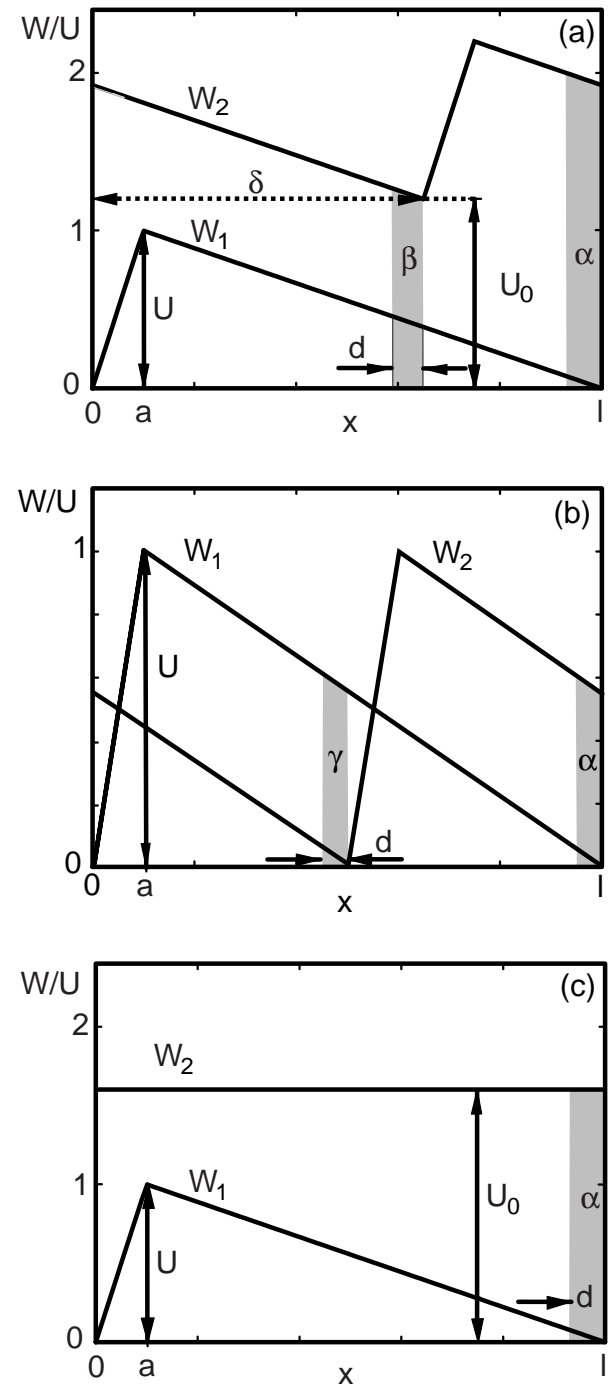

Figure 2. Three choices of potentials $W_{1}$ and $W_{2}$ with period $l$ and transition regions indicated in grey. The position $a$ of the maximum of $W_{1}$ characterizes the potential asymmetry, $U$ denotes the potential amplitudes. (a) System A with potentials shifted by a distance $\delta$ and offset $U_{0}$. Active transitions $\alpha$ and thermal transitions $\beta$ are localized within regions of size $d$ near the potential minima. (b) System B with symmetric states. The potentials are shifted by a distance of $l / 2$, active transitions $\alpha$ and $\gamma$ are chosen such that the system is symmetric with respect to an exchange of the two states. (c) System $\mathrm{C}$ with a flat potential $W_{2}$, localized active transitions $\alpha$ and non-localized thermal transitions $\beta$. 


\section{EFFICIENCIES CLOSE TO AND FAR FROM EQUILIBRIUM}

\section{A. Specific examples}

We have introduced a general framework which allows us to study a large variety of systems which differ in their potential shapes and in the transition rates $\alpha_{2}, \gamma_{2}$ and $\beta_{2}$. We now discuss three particular examples which we have chosen as prototypes to illustrate the physics of energy transduction.

System A is a system with two periodic potentials of equal amplitude $U$ which are piecewise linear and which are shifted with respect to each other by a displacement $\delta$ as shown schematically in Fig. 2 (a). Furthermore, they differ by a constant value $U_{0}: W_{2}(x)=W_{1}(x-\delta)+U_{0}$. The potentials are characterized by the asymmetry parameter $a$ which denotes the position of the potential maximum of $W_{1}$. We choose a reaction scheme with chemically activated transitions $\alpha_{1,2}$ between the low energy state 1 and the high energy state 2 , passive transition $\beta_{1,2}$ and $\gamma_{1,2}=0$. The chemical cycle corresponds to subsequent transitions $\alpha$ and $\beta$ which we choose localized within intervals of size $d$ :

$$
\alpha_{2}(x)= \begin{cases}\omega & l-d \leq x \leq l \\ 0 & \text { otherwise }\end{cases}
$$

localized near the minimum of $W_{1}$ and $\beta_{2}(x)=\alpha_{2}(x-\delta)$ localized near the minimum of $W_{2}$, see Fig. 2 (a). Here we have for simplicity introduced a single parameter $\omega$ which sets the typical time scale of transition rates. The transition rates $\omega_{\sigma}$ of system A obey

$$
\begin{array}{ll}
\omega_{1}(x)=\alpha_{2}(x) e^{\left(W_{1}-W_{2}+\Delta \mu\right) / T} & +\alpha_{2}(x-\delta) e^{\left(W_{1}-W_{2}\right) / T} \\
\omega_{2}(x) & =\alpha_{2}(x) \\
& +\alpha_{2}(x-\delta)
\end{array}
$$

System A is chosen in such a way that diffusion within the potentials is not necessary for motion generation and each chemical cycle generates with high probability a forward step along the $x$-coordinate.

System B has different symmetry and different topology of the chemical reaction scheme as compared to system A, see Fig 2 (b). The two potentials are shifted by exactly half a potential period $\delta=l / 2: W_{2}=W_{1}(x-l / 2)$ and $U_{0}=0$. This allows us to introduce a new symmetry: the system is invariant under a shift $x \rightarrow x+l / 2$ if at the same time the states are exchanged: $1 \rightarrow 2$. This situation is realized by choosing transition rates $\beta_{1,2}=0$ and $\gamma_{1}(x)=\alpha_{2}(x-l / 2)$ where we localize all transitions near the potential minima. We can therefore write for system B

$$
\begin{aligned}
& \omega_{1}(x)=\alpha_{2}(x) e^{\left(W_{1}-W_{2}+\Delta \mu\right) / T}+\alpha_{2}(x-l / 2) \\
& \omega_{2}(x)=\alpha_{2}(x)+\alpha_{2}(x-l / 2) e^{\left(W_{2}-W_{1}+\Delta \mu\right) / T},
\end{aligned}
$$

with $\alpha_{2}(x)$ given by Eq. (36). Note, that system B involves two active chemical steps per potential period.
However, because of its additional symmetry it is $l / 2$ periodic. Furthermore, all chemical transitions involve ATP hydrolysis, there are no passive transitions.

System $\mathbf{C}$ is shown in Fig. 2(c). It is a variant of model A with a weakly bound state $W_{2}(x)=U_{0}$ of constant energy. As for system A we choose a reaction scheme with $\gamma_{1,2}=0$ and localized active transitions near the minima using again definition (36). Since the potential $W_{2}$ is structureless, we assume passive transitions to be non-localized with $\beta_{2}(x)=\omega$. Therefore in system $\mathrm{C}$

$$
\begin{array}{ll}
\omega_{1}(x)=\alpha_{2}(x) e^{\left(W_{1}-W_{2}+\Delta \mu\right) / T}+\omega e^{\left(W_{1}-W_{2}\right)} \\
\omega_{2}(x)=\alpha_{2}(x) & +\omega .
\end{array}
$$

In this case motion generation involves a diffusive step in state 2 which we expect to reduce the efficiency of energy transduction.

In order to discuss these models, we identify the relevant dimensionless parameters: the dimensionless position $\bar{x}=x / l$, reduced temperature $t=T / U$, reduced potentials $w_{\sigma}=W_{\sigma} / U-f_{\text {ext }} l / U$ and reduced transition rates $\bar{\omega}_{\sigma}=\omega_{\sigma} / \omega$. Eqns. (13) and (14) can for a steady state be written as

$$
\begin{aligned}
-\partial_{\bar{x}}\left(t \partial_{\bar{x}} P_{1}+P_{1} \partial_{\bar{x}} w_{1}\right) & =\chi\left(-\bar{\omega}_{1} P_{1}+\bar{\omega}_{2} P_{2}\right) \\
-\partial_{\bar{x}}\left(t \partial_{\bar{x}} P_{2}+P_{2} \partial_{\bar{x}} w_{2}\right) & =\chi\left(\bar{\omega}_{1} P_{1}-\bar{\omega}_{2} P_{2}\right) .
\end{aligned} .
$$

The dimensionless parameter

$$
\chi \equiv \frac{\omega \xi l^{2}}{U}
$$

compares two time-scales: (i) the typical chemical time $\omega^{-1}$ and (ii) the typical sliding time in the potentials $\xi l^{2} / U$. For $\chi \gg 1$ transitions are fast compared to sliding while for $\chi \ll 1$ sliding is fast. The model is fully characterized by the dimensionless parameters $\chi, T / U$, $\Delta \mu / U, a / l, d / l, \delta / l$ and $U_{0} / U$. The results discussed in the following section are obtained by numerically solving Eq. (40) with periodic boundary conditions for the three different systems.

\section{B. Efficiencies close to equilibrium}

Numerical examples for the maximal efficiency in the linear response regime as a function of temperature are displayed in Fig. 3 for systems A, B and C and different values of $\chi=\omega \xi l^{2} / U$. They have been obtained by first calculating Onsager coefficients from steady state solutions for small $\Delta \mu$ and small $f_{\text {ext }}$ and using Eq. (12). The orders of magnitude of the efficiency differ for systems A, B and C. The efficiency $\eta$ depends on $\chi$ and increases in general with increasing $\chi$. System B has the largest efficiency which approaches $\eta \simeq 1$ for small $T / U$ and decreases monotonically as a function of temperature. For systems $\mathrm{A}$ and $\mathrm{C}$ the efficiency has a maximum as a function of temperature and vanishes in the limit of 
small $T / U$. This indicates in these cases the importance of thermal fluctuations for energy transduction. Note, that the limit of small temperatures is subtle since in linear response $\Delta \mu \ll T$ must be obeyed. Therefore, this limit corresponds to first sending $\Delta \mu$ to zero and $T$ afterwards. Even for small temperatures the system thus remains in a regime where thermally activated passage over energy barriers rests important. While system A can have significant efficiencies of the order of $\eta \simeq 0.06$ in the linear response regime, the efficiency of system $\mathrm{C}$ which relies on diffusive steps is small $\left(\eta \simeq 10^{-4}\right)$, see Fig. 3.
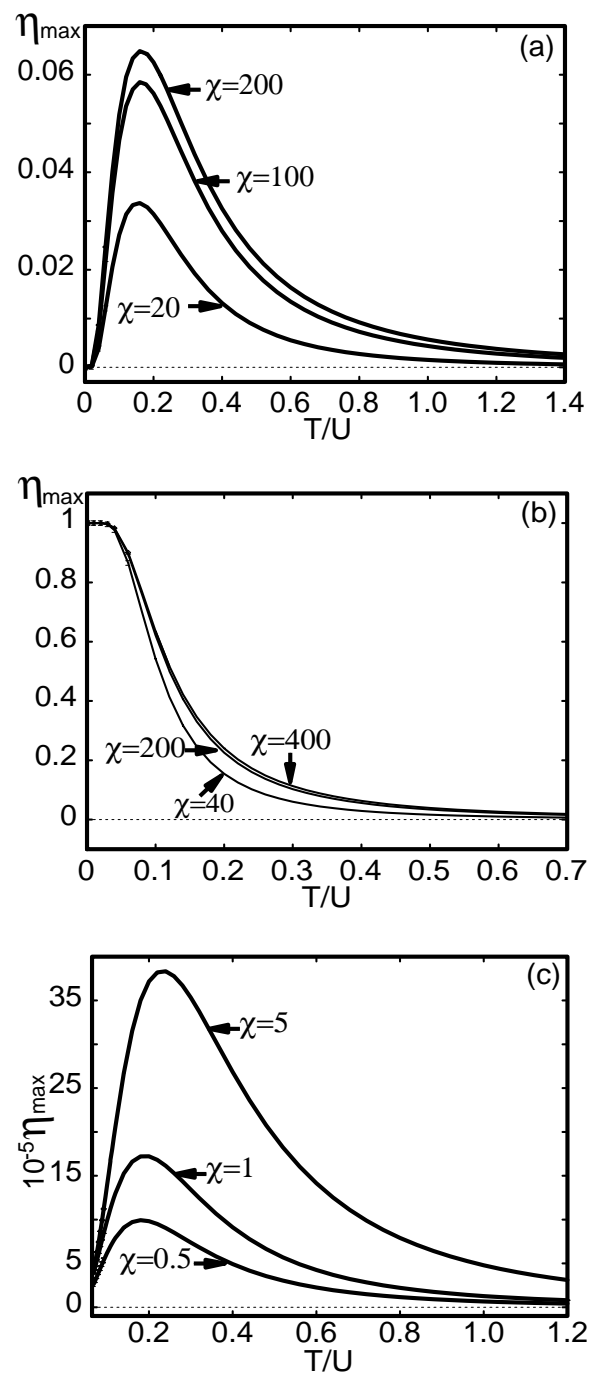

Figure 3. Maximal efficiency $\eta_{\max }$ in the linear response regime as function of reduced temperature $T / U$ for systems $\mathrm{A}, \mathrm{B}$ and $\mathrm{C}$ with $a / l=0.1$ as shown in Fig. A. (a) System A with $\delta / l=0.65, U_{0} / U=0.4$, at different $\chi$. (b) Same diagram for system B at different $\chi$. (c) Same diagram for system $\mathrm{C}$ with $U_{0} / U=1.2$ at different $\chi$.
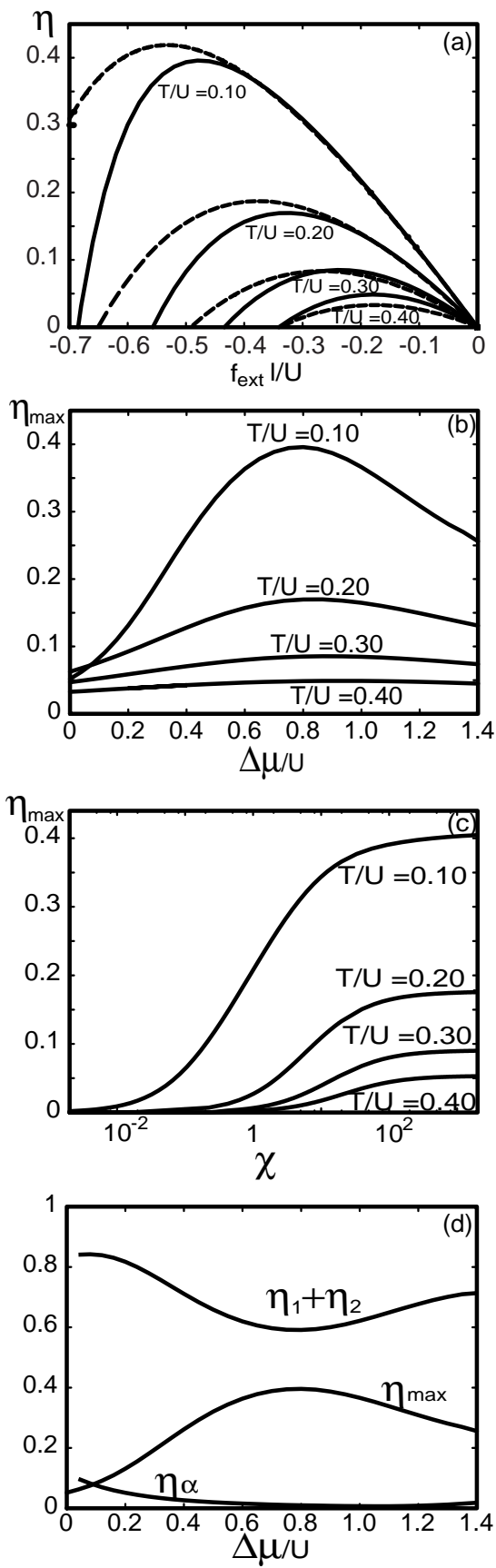

Figure 4. Energy transduction of system (A) with $a / l=0.1$ and $U_{0} / U=0.4$. (a) Efficiency $\eta$ as a function of the external force $f_{\text {ext }}$ for $\Delta \mu / U=0.8, \chi=\xi l^{2} \omega / U=200$, and different reduced temperatures $T / U$. Broken lines represent the approximation discussed in the text. (b) Maximal efficiency $\eta_{\max }$ as a function of $\Delta \mu / U$ for $\chi=200$ and different temperatures. (c) Maximal efficiency as a function of $\chi$ for $\Delta \mu / U=0.8$ and different temperatures. (d) Relative dissipation rates as a function of $\Delta \mu$ for the same system: shown are the fraction of energy dissipated by potential sliding $\eta_{1}+\eta_{2}$ and the fraction dissipated via active transitions $\eta_{\alpha}$. For details see text. 


\section{Efficiencies far from equilibrium}

We have shown that the two state model transduces chemical energy into mechanical work in the linear response regime, however with varying efficiencies. In linear response the chemical action represents a small bias of the dominant thermal fluctuations. We are now comparing these results with the properties of energy transduction far from equilibrium.

System A: Fig. 17 (a) displays the efficiency $\eta$ as a function of the applied force for the system A as defined in Fig. 2 for $\Delta \mu / T=8$ and different temperatures. The efficiency vanishes for $f_{\text {ext }}=0$ as well as for the stall force for which the velocity vanishes. For an intermediate value of the force, the efficiency reaches a maximum. This value $\eta_{\max }$ is displayed in Fig. \# (b) as a function of $\Delta \mu$. This diagram reveals the main characteristics of energy transduction: for small $\Delta \mu$ we find again the nonvanishing efficiency of the linear regime. The efficiency increases as a function of $\Delta \mu$, reaches a maximal value and decreases for large $\Delta \mu$ to zero. For sufficiently large values of $\Delta \mu$ the efficiency increases for decreasing temperatures and reaches in the example shown a value of $\eta \simeq 0.4$ for $T / U \simeq 0.1$. The results obtained for different temperatures intersect for small $\Delta \mu$ which corresponds to the observation discussed above that the efficiency in the linear regime displays a maximum as a function of temperature. Fig. A(c) shows the behavior of $\eta_{\max }$ for fixed $\Delta \mu / U$ as a function of $\chi=\xi l^{2} \omega / U$ over a range of 6 decades. The efficiency increases monotonically with increasing $\chi$ from zero to a plateau value. As an important result we find that the largest values of the efficiency for the relevant energy scale $T / U \simeq 0.1$ are of the order of $\eta \simeq 0.5$ and occur for $\Delta \mu \sim U$ comparable to the energy difference between the two states at the transition and thus far from the linear regime.

The dissipation rate $\Pi$ can, according to Eq. 35, be divided into separate contributions of potential sliding $\Pi_{\sigma}$ and chemical transitions $\Pi_{\mu}$, with $\mu=\alpha, \beta, \gamma$. It is useful to define relative dissipation rates $\eta_{\sigma}=\Pi_{\sigma} / r \Delta \mu$ and $\eta_{\alpha}=\Pi_{\alpha} / r \Delta \mu$ which are analog to the efficiency and describe the fraction of dissipated energy relative to the consumed chemical work. Note that $\eta+\eta_{1}+\eta_{2}+\eta_{\alpha}+$ $\eta_{\beta}+\eta_{\gamma}=1$ follows from energy conservation. Fig. 田 (d) shows the dominant relative dissipation rates together with the efficiency $\eta$. The dominant dissipation is $\Pi_{1}+$ $\Pi_{2}$ resulting from potential friction, dissipation $\Pi_{\alpha}$ of chemical transitions, plays a minor role. The dissipation $\eta_{\beta}$ corresponding to passive transitions is smaller than 0.01 and can be neglected. It is therefore not shown. The maximum of $\eta$ corresponds to a minimum of $\eta_{1}+\eta_{2}$.

The main energy loss results from thermally activated backward steps. This idea can be directly tested by calculating the local dissipation density $\Theta_{1}(x)+\Theta_{2}(x)$ as defined in Eq. (28). This quantity is displayed in Fig. 5. The plot reveals that maximal dissipation occurs for $\delta-a<x<\delta$, i.e. along the steep potential slope of the potential $W_{2}$.

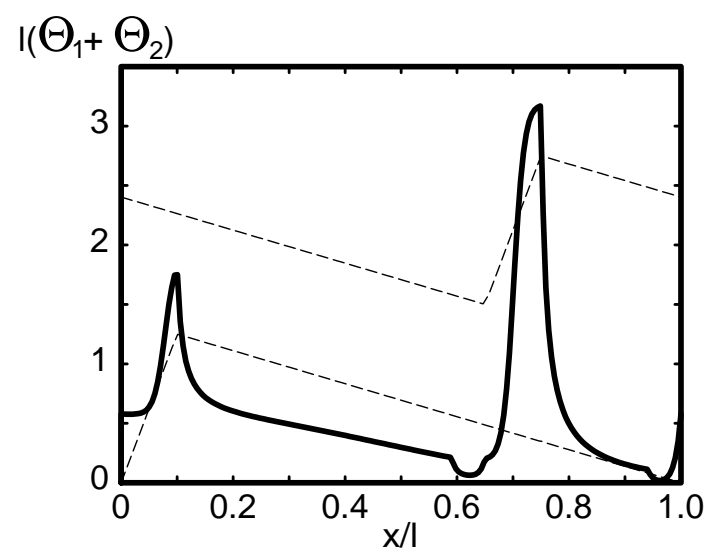

Figure 5. Density $\Theta_{1}(x)+\Theta_{2}(x)$ as defined in Eq. (28) of the dissipation rate as a function of position $x$ for system A with $U l / f_{\text {ext }}=-0.48, \chi=200, \Delta \mu / U=0.80$.

A second maximum of local dissipation exists for $0<$ $x<a$ along the steep slope of $W_{1}$. In contrast, minimal dissipation occurs near the potential minima where transitions between states take place. The steep potential slopes where the density of energy dissipation is large indeed are accessed via thermally activated backward hopping events. The probability of such events increases in the presence of an "adverse" external force which limits the efficiency of the system.

System B: Fig. 6 displays the same information for system B. The diagram reveals that efficiencies are in general larger than for system $\mathrm{A}$, reaching values up to $\eta \simeq 0.7$ for $T / U=0.05$. Furthermore, the maximum of the efficiency as a function of $\Delta \mu$ is less pronounced and shifted to small values of $\Delta \mu$ as compared to system A. One might expect that the dissipation due to passive transitions $\eta_{\beta}$ in system A which does not exist in system B could play a role in improving the efficiency of system B. However as discussed above $\eta_{\beta}$ can be neglected and is thus not responsible for this effect. The main reason for the improved efficiency of system B is the fact that the effective energy barrier for thermally activated passage over the potential maxima is larger in system B as compared to system A. Therefore, fluctuations leading to "backward steps" in the opposite direction of average motion which completely dissipate a consumed ATP molecule are less likely. Each active chemical transition is thus transduced into work with high probability. Fig. 6 (c) shows qualitatively the same behavior of the efficiency as a function of $\chi$ for system B as compared to system A. Also as discussed for system A, the dominant dissipation process corresponds to sliding in the potentials, see Fig.6(d). Note that the efficiency is larger than in system A, which correlates with the increased barrier height reducing the probability of backward steps.

System C: Energy transduction of system C which 
involves diffusive steps and non-localized de-excitations.
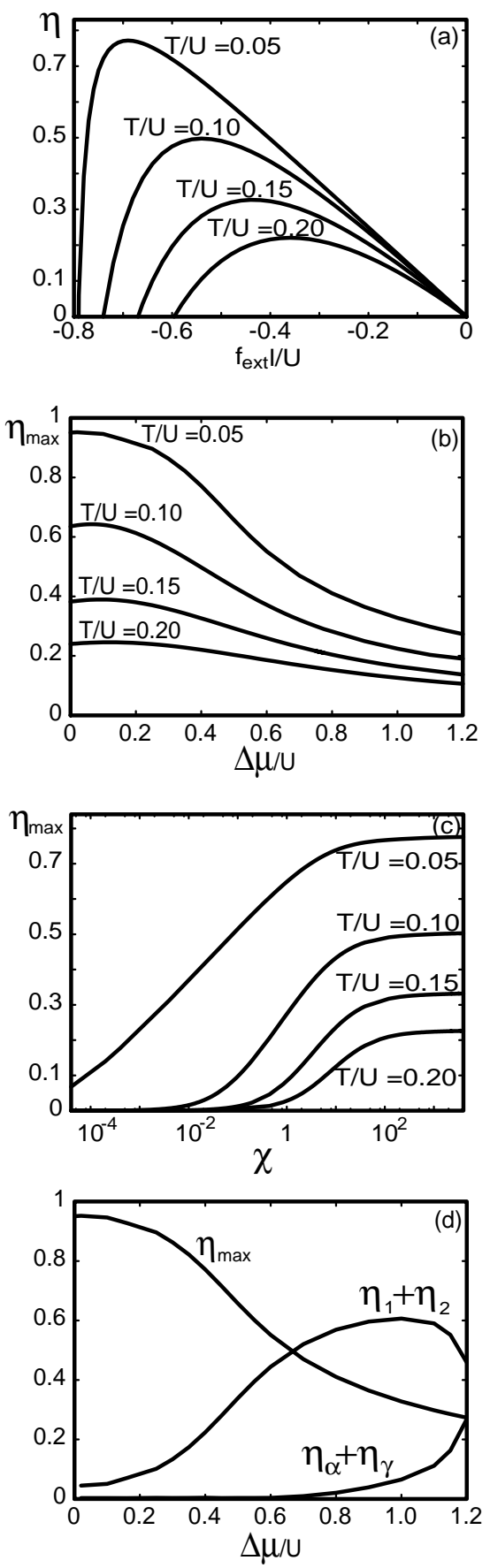

Figure 6. Energy transduction of system B with $a / l=0.1$. (a) Efficiency $\eta$ as a function of the external force $f_{\text {ext }}$ for $\Delta \mu / U=0.4, \chi=\xi l^{2} \omega / U=400$, and different reduced temperatures $T / U$. (b) Maximal efficiency $\eta_{\max }$ as a function of $\Delta \mu / U$ for $\chi=400$ and different temperatures. (c) Maximal efficiency as a function of $\chi$ for $\Delta \mu / U=0.4$ and different temperatures. (d) Relative dissipation rates as a function of $\Delta \mu$ : the fraction of energy dissipated by potential sliding $\eta_{1}+\eta_{2}$ and the fraction dissipated via active transitions $\eta_{\alpha}+\eta_{\gamma}$.

Maximal efficiencies are of the order of 0.02 and thus much smaller than those for systems A and B, see Fig. 7. As in system $\mathrm{A}$ the largest efficiencies occur for $\Delta \mu \gg T$ and thus far from equilibrium. The reason for the reduced efficiency becomes clear when studying the relative dissipation rates shown in Fig. 7 (b): Most energy is in this case dissipated by the passive and active transitions, potential sliding is less important. In particular, the nonlocalized and passive de-excitations dominate dissipation far from equilibrium.
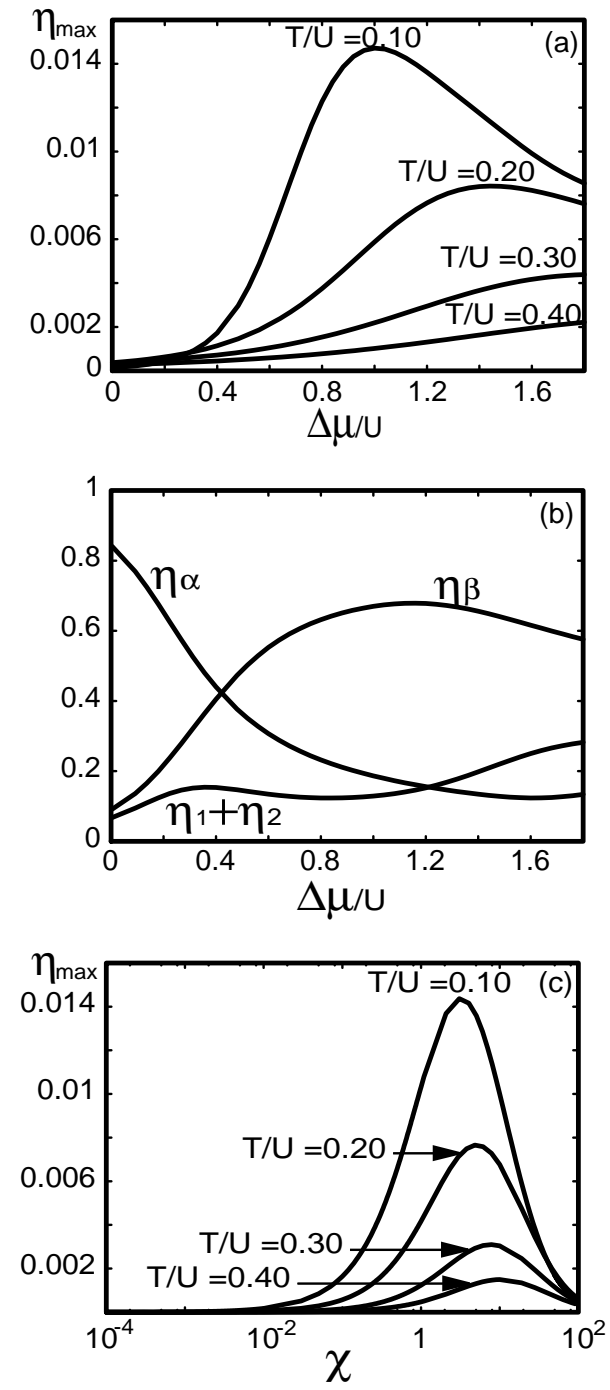

Figure 7. Energy transduction of system C with $a / l=0.1$. (a) Maximal efficiency $\eta$ as a function of $\Delta \mu / U$ for $\chi=\xi l^{2} \omega / U=5, U_{0} / U=1.2$ and different reduced temperatures $T / U$. (b) Relative dissipation $\eta_{1}+\eta_{2}$ in the potentials as well as the dissipation of transitions $\eta_{\alpha}$ and $\eta_{\beta}$ corresponding to (a). (c) Maximal efficiency as a function of $\chi$ for $\Delta \mu / U=1.2$ and different temperatures.

Very striking is the behavior of the efficiency as a function of $\chi$ shown in Fig. 7 (c): The efficiency displays a maximum for certain values of $\chi$ but vanishes both for 
large and small $\chi$. This property reflects the fact that a matching of time scales is crucial for this system: The life-time in the excited state should be comparable to the diffusion-time over a potential period:

$$
l^{2} \sim T / \xi \omega
$$

Therefore, the optimal value of $\chi$ should behave as $\chi_{\mathrm{opt}} \sim$ $T / U$ which explains the temperature-dependence of the maximum in Fig. 7 (c).

\section{Approximation for small forces}

The efficiency far from equilibrium for $\Delta \mu / T \gg 1$ but for small forces can be understood by a simple approximation which we discuss for system A. In the limit of large $\Delta \mu$ and $U / T$ we ignore spontaneous hopping events over the maxima of potential $W_{1}$. Every ATP consumption event corresponds to a transition to the second state from which the particle will eventually decay to the first state. During this process, it undergoes a forward step with probability $p_{+}$, a backward step with probability $p_{-}$ or it will return to the initial position with probability $p_{0}$. Here, we have ignored multiple steps, see Fig. 8.

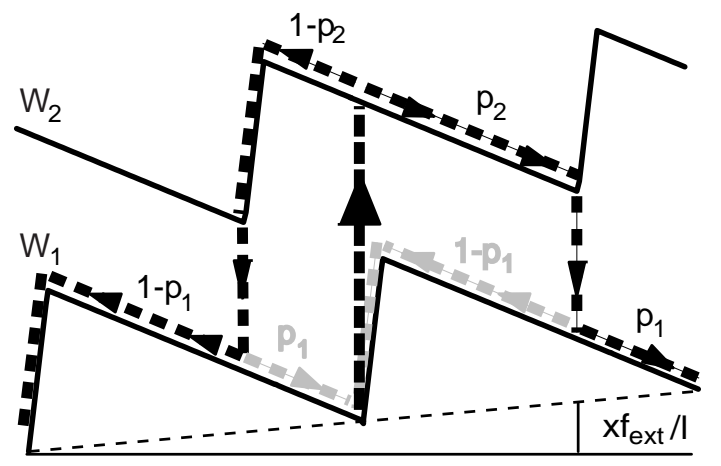

Figure 8. Schematic diagram of events after consumption of one fuel molecule. Forward steps occur with probability $p_{+}=p_{1} p_{2}$, backward steps with probability $p_{-}=\left(1-p_{2}\right)\left(1-p_{1}\right)$ and neutral steps with $p_{0}=p_{2}\left(1-p_{1}\right)+p_{1}\left(1-p_{2}\right)$.

In the presence of an external force $f_{\text {ext }}$, the efficiency can thus be estimated as

$$
\eta \simeq-\frac{f_{\text {ext }}}{\Delta \mu}<x>
$$

where

$$
<x>\simeq l\left(p_{+}-p_{-}\right) \simeq v / r
$$

is the average displacement per consumed ATP. The probabilities $p_{ \pm}$can be written as

$$
p_{+}=p_{2}(0) p_{1}(\delta) \quad, \quad p_{-}=\left(1-p_{2}(0)\right)\left(1-p_{1}(\delta)\right) \quad .
$$

Here, we have introduced the probabilities $p_{\sigma}(x)$ for motion in the forward direction after a particle appears in state $\sigma$ at position $x$. Similarly, $1-p_{\sigma}(x)$ is the probability for backward motion, see Fig. 8. Since the two potentials are shifted with respect to each other $p_{2}(x)=p_{1}(x-\delta)$. The probability $p_{+}$requires two subsequent forward movements of this type, $p_{-}$results from two backward movements. As described in appendix B, the probabilities $p_{\sigma}(x)$ can be calculated approximatively for large $U / T$. Fig. 4 (a) shows the efficiency estimated by Eqns. (44) and (45) together with the numerically obtained values for comparison. For small forces the agreement is good, thus confirming our simplified picture of energy transduction in this regime.

\section{CONCLUDING REMARKS}

In the previous sections, we have studied the efficiency of energy transduction from chemical energy to mechanical work using a simple two-state model under isothermal conditions. We considered three different examples: system A with two shifted potentials and both active and thermal transitions between the two states localized at the potential minima; system $\mathrm{B}$ with an additional symmetry between the two states and no passive thermal transitions; and finally system $\mathrm{C}$ with a flat weakly bound state and non-localized passive transitions. We demonstrated that energy transduction can be very efficient in the systems $\mathrm{A}$ and $\mathrm{B}$ with localized transitions and shifted potentials and is at least two orders of magnitude smaller in system $\mathrm{C}$ which requires diffusive steps for motion to occur. Interestingly, the largest efficiency can occur far from equilibrium. This is in particular the case for systems $\mathrm{A}$ and $\mathrm{C}$ which both are not very efficient in the linear response regime.

\section{A. Isothermal motors, heat engines and Brownian ratchets}

Efficiencies of energy transduction have been studied and discussed for a long time. Of particular significance is the concept of Carnot which defines the efficiency of macroscopic heat engines coupled to two thermal baths at temperatures $T^{ \pm}$and $T^{+}>T^{-}$as

$$
\eta_{\text {Carnot }}=-\frac{f_{\text {ext }} v}{\dot{Q}^{+}},
$$

where $\dot{Q}^{+}$is the rate of heat transfer from the hot reservoir. This definition then leads to an upper limit of the efficiency $\eta_{\text {Carnot }} \leq\left(T^{+}-T^{-}\right) / T^{+}$which cannot be surpassed by any heat engine. In order to characterize energy transduction in biological systems, a natural choice is 23

$$
\eta=-\frac{f_{\mathrm{ext}} v}{r \Delta \mu},
$$


which we have adopted in this paper, see Eq. (6), and which is based on the chemical potential difference between fuel and reaction products. As we have discussed, this efficiency obeys $\eta \leq 1$ in order to satisfy the first law of thermodynamics, but there is no nontrivial upper bound. In addition to the obvious fact that $\eta_{\text {Carnot }}$ describes a heat engine and $\eta$ an isothermal motor, there remains a fundamental difference between the two cases: the definition of $\eta_{\text {Carnot }}$ assumes that all heat dissipated in the bath $T^{-}$is lost. This is true in most practical cases, however if the bath at $T^{-}$was also used as the hot bath of a second heat engine, some of this energy could in principle be reused. Similarly, the definition of $\eta$ takes into account the energy of the lower-energy reservoir, thus assuming that the energy of the reaction products remains available. One might think that it is possible to avoid this difference between the two definitions by choosing:

$$
\eta^{\prime}=-\frac{f_{\mathrm{ext}} v}{r \mu_{A}}
$$

where $\mu_{A}$ would be the chemical potential of the fuel (ATP). This definition would share with Carnot's definition the viewpoint that the energy of the reaction products are not useful, and since $\eta^{\prime}=\eta \Delta \mu / \mu_{A}$ would lead to the upper bound $\eta^{\prime} \leq\left(\mu_{A}-\mu_{P}\right) / \mu_{A}$. Such a choice, however, suffers from a serious shortcoming: only chemical potential differences are physically meaningful. Depending on the state of reference used for defining $\mu_{A}$, the value of $\mu_{A}$ could be positive, negative or even vanish.

The example given above demonstrates that comparing efficiencies can be dangerous as they may be based on different definitions corresponding to different points of view. This is also the case for ratchet models which have been studied in many variants and under widely varying physical conditions. All definitions described above have been used in the literature: The definition $\eta_{\text {Carnot }}$ for systems driven by temperature differences [9, 12, 14, 16, 17], the definition for $\eta$ given by Eq. (6) 23, 8 , 15 as well as $\eta^{\prime}$ 13. Alternative definitions have been proposed for situations where the chemical reaction is not fully specified [11,18]. Other definitions of energy transduction efficiencies have been used for systems which are driven by stochastic or deterministic forces 12,19 21]. Recently, Sekimoto has presented a unified picture which includes most systems in a common framework [12]. However, in general, a given definition is adapted to one particular physical situation.

\section{B. The two-state model and biological motors}

One important motivation of this work is to clarify the general properties of energy transduction of biological motors. The characteristic behaviors of our system $\mathrm{A}$ and $\mathrm{B}$ with localized transitions and shifted potentials are similar to those observed for processive biological motors such as e.g. kinesins which move along microtubules and for which the consumption of ATP and the subsequent stepping are strongly correlated for small external forces 29, 31, 32. Kinesin motors consist of two identical active head groups which both hydrolize ATP [33]. There is evidence suggesting that the motor could "walk" in a head-over-head fashion along microtubules, detaching a head in the back and reattaching in front of the molecule while keeping the second head bound [34,35. In such a picture each ATP-hydrolysis cycle leads to a new situation where both heads have exchanged their roles and the center of mass of the molecule has advanced one filament period. This type of motion is captured in a simple way in the variant B of our model which is symmetric with respect to the two states. Because of this symmetry, both states are indistinguishable but the corresponding potentials are shifted by $l / 2: W_{2}(x)=W_{1}(x-l / 2)$. We therefore identify each of the two states with one kinesin head and $l / 2$ with the filament period, see Fig. 2(b). Recently, system $\mathrm{C}$ with a structureless excited state has been used for single kinesin heads which were observed to move processively along a microtubule [36]. Models of this type have typically been considered in the context of nonprocessive motors such as myosins which have a weakly bound state during their interaction cycle. Myosins interact with a filament to generate displacements of the order of several nm, but they do not continuously move along a filament as individual motors since they easily lose their track and diffuse away [37,38. The latter phenomenon is not captured in the one-dimensional two-state model, however the flat potential of system $\mathrm{C}$ requires diffusive steps for average motion and the efficiency is therefore smaller than for systems A and B. Under physiological conditions non-processive motors operate not as isolated enzymes but move together in large groups. In this situation, however, diffusive steps become unimportant and the efficiency becomes large and reaches the same orders of magnitude as for model A and B described here 39, 40,11 .

When comparing our simple models with biological motors, the value of the adimensional parameter $\chi=$ $\omega \xi l^{2} / U$ introduced in Eq. (41) is crucial. The relevant orders of magnitude for most parameters are well known: Energy scales are $U \simeq 10 T$ [23], typical time scales of conformational changes are $\omega^{-1} \simeq 1 \mathrm{~ms}$ and the relevant length scale is $l \simeq 5-10 \mathrm{~nm}$ [37. However, the friction coefficient $\xi$ is unknown and difficult to estimate. Therefore, we do not know at which value of $\chi$ biological motors operate. The role of $\chi$ on the functioning of the system can be discussed by comparing both the maximal efficiency and the dimensionless velocity $v / \omega l$ as a function of $\chi$, see Fig. 9. The diagram reveals that for large values of $\chi$ for which the efficiency is large the velocities become small. For small $\chi$ velocities are optimal but efficiency becomes negligible. This observation suggests that optimal conditions are obtained in the intermediate regime $\chi \simeq 0.1-1$ where chemical times and sliding times along the potential slopes become comparable. If linear molecular motors operate in this regime, the microscopic 
friction coefficient $\xi$ is of the order of $10^{-7}-10^{-6} \mathrm{~kg} / \mathrm{s}$. If we estimate $\xi$ from simple hydrodynamic arguments $\left(\xi_{\mathrm{h}} \simeq 6 \pi \eta_{\mathrm{vis}} l\right)$, where $\eta_{\mathrm{vis}}$ is some measure of a "local" viscosity, we find $\eta_{\text {vis }} \simeq 10-100$ Poise, $10^{3}-10^{4}$ times the viscosity of water, values compatible with dense macromolecular solutions. Interestingly, this order of magnitude, corresponds to a diffusion coefficient of $4 \cdot 10^{-14}$ $\mathrm{m}^{2} / \mathrm{s}$, a value reported recently for single headed kinesin [36. This observation together with our estimate suggest that linear molecular motors are optimized both from the velocity and the efficiency standpoint.

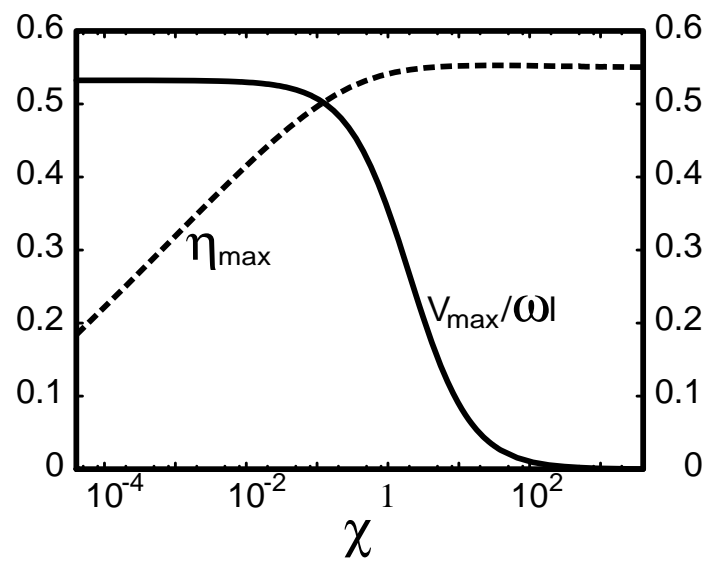

Figure 9. Maximal efficiencies (broken line) and normalized velocities (solid line) as a function of $\chi$ for system B and $\Delta \mu / U=0.6$ and $T / U=0.05$.

\section{ACKNOWLEDGMENTS}

We acknowledge stimulating discussions with S. Camalet, R. Everaers, P.G. de Gennes, K. Sekimoto and T. Shibata.

\section{APPENDIX A: ONSAGER COEFFICIENTS}

In linear response theory, the behavior of the system is completely defined by the Onsager coefficients $\lambda_{i j}$. The Onsager symmetry relation (10) follows from general thermodynamic arguments and the microscopic reversibility. The calculation of Onsager coefficients is difficult, however the symmetry relation can be verified by general arguments as shown in section A1. In section A 2 we obtain explicit expressions for the coefficients $\lambda_{i j}$ for a many-motor system as introduced in 8,11.

\section{Symmetry relation for a single motor}

In order to demonstrate the symmetry relation of Onsager coefficients for the two-state model, we start from the probability distributions at equilibrium $\left(f_{\text {ext }}=0\right.$, $\Delta \mu=0)$ as

$$
P_{\sigma}^{e q}=N e^{-W_{\sigma}(x) / T}
$$

with a normalization factor

$$
N^{-1} \equiv \sum_{\sigma} \int_{0}^{l} d x e^{-W_{\sigma} / T} .
$$

For small $f_{\text {ext }} l / T \ll 1$ and $\Delta \mu / T \ll 1$ we define deviations $p_{\sigma}(x)$ from equilibrium which obey

$$
P_{\sigma}(x)=N e^{-W_{\sigma} / T}\left(1+p_{\sigma}(x)\right)
$$

Without loss of generality we consider the case where only the transitions $\alpha_{\sigma}$ and $\beta_{\sigma}$ occur but $\gamma_{\sigma}=0$. To linear order in $\Delta \mu$, the transition rates can be written as

$$
\begin{aligned}
& \omega_{1}(x)=\left(\omega(x)+\frac{\alpha(x) \Delta \mu}{T}\right) e^{W_{1} / T} \\
& \omega_{2}(x)=\omega(x) e^{W_{2} / T}
\end{aligned}
$$

with $\alpha=\alpha_{2} e^{-W_{2} / T}$ and $\omega(x) \equiv\left(\alpha_{2}+\beta_{2}\right) e^{-W_{2} / T}$. Using Eq. (13) we find to linear order

$$
\begin{aligned}
& -\frac{T}{\xi} \partial_{x}\left(e^{\frac{-W_{1}(x)}{T}} \partial_{x} p_{1}\right)+\omega(x)\left(p_{1}-p_{2}\right)=h_{1}(x) \\
& -\frac{T}{\xi} \partial_{x}\left(e^{\frac{-W_{2}(x)}{T}} \partial_{x} p_{2}\right)-\omega(x)\left(p_{1}-p_{2}\right)=h_{2}(x) .
\end{aligned}
$$

The fields $h_{\sigma}(x)$ are nonzero in the presence of mechanical or chemical forces:

$$
\begin{aligned}
& h_{1}(x)=-\alpha(x) \Delta \mu / T-\partial_{x} e^{-\frac{W_{1}(x)}{T}} f_{\text {ext }} / \xi \\
& h_{2}(x)=\alpha(x) \Delta \mu / T-\partial_{x} e^{-\frac{W_{2}(x)}{T}} f_{\text {ext }} / \xi .
\end{aligned}
$$

Eq. (A5) represents a linear relation between $p_{\sigma}$ and $h_{\sigma}$ which can be inverted and which thus defines a response kernel

$$
p_{\sigma}(x)=\sum_{\rho} \int_{0}^{l} d x^{\prime} \chi_{\sigma \rho}\left(x, x^{\prime}\right) h_{\rho}\left(x^{\prime}\right) .
$$

This allows us to express the velocity and the fuel consumption rate within linear response theory:

$$
\begin{aligned}
v= & \int_{0}^{l} d x\left[\xi^{-1} e^{-\frac{W_{1}}{T}}\left(f_{\text {ext }}-T \partial_{x} p_{1}\right)+\right. \\
& \left.+\xi^{-1} e^{-\frac{W_{2}}{T}}\left(f_{\text {ext }}-T \partial_{x} p_{2}\right)\right] \\
r= & \int_{0}^{l} d x \alpha(x)\left[\left(p_{1}-p_{2}\right)+\Delta \mu / T\right]
\end{aligned}
$$

The Onsager coefficients $\lambda_{i j}$ can be written in terms of the response functions $\chi_{\sigma \rho}\left(x, x^{\prime}\right)$. In particular, we find for the coefficients of mechano-chemical coupling 


$$
\begin{aligned}
\lambda_{12} & \equiv \frac{\partial v}{\partial \Delta \mu} \\
& =\int_{0}^{l} d x \int_{0}^{l} d x^{\prime}\left[\xi^{-1} e^{-\frac{W_{1}(x)}{T}}\left(\partial_{x} \chi_{11}\left(x, x^{\prime}\right)-\partial_{x} \chi_{12}\left(x, x^{\prime}\right)\right)\right. \\
& \left.+\xi^{-1} e^{-\frac{W_{2}(x)}{T}}\left(\partial_{x} \chi_{21}\left(x, x^{\prime}\right)-\partial_{x} \chi_{22}\left(x, x^{\prime}\right)\right)\right] \alpha\left(x^{\prime}\right)
\end{aligned}
$$

$$
\begin{aligned}
\lambda_{21} & \equiv \frac{\partial r}{\partial f_{\mathrm{ext}}} \\
& =-\int_{0}^{l} d x \int_{0}^{l} d x^{\prime}\left[\left(\chi_{11}\left(x, x^{\prime}\right)-\chi_{21}\left(x, x^{\prime}\right)\right) \xi^{-1} \partial_{x^{\prime}} e^{-\frac{W_{1}\left(x^{\prime}\right)}{T}}\right. \\
& \left.+\left(\chi_{12}\left(x, x^{\prime}\right)-\chi_{22}\left(x, x^{\prime}\right)\right) \xi^{-1} \partial_{x^{\prime}} e^{-\frac{W_{2}\left(x^{\prime}\right)}{T}}\right] \alpha(x) .
\end{aligned}
$$

Performing a partial integration in Eq. A10, we find that the Onsager symmetry relation (10) is satisfied exactly if the response functions obey the symmetry relation

$$
\chi_{\sigma \rho}\left(x, x^{\prime}\right)=\chi_{\rho \sigma}\left(x^{\prime}, x\right) .
$$

This symmetry relation follows from the hermiticity of the linear operator $\mathcal{L}$ defined in Eq. A5 which can be expressed as

$$
\mathcal{L}\left(\begin{array}{l}
p_{1} \\
p_{2}
\end{array}\right)=\left(\begin{array}{l}
h_{1} \\
h_{2}
\end{array}\right)
$$

where

$$
\mathcal{L}=\left(\begin{array}{cc}
\mathcal{L}_{1}+\omega & -\omega \\
-\omega & \mathcal{L}_{2}+\omega
\end{array}\right)
$$

with

$$
\mathcal{L}_{i}=-\xi^{-1} T \partial_{x} e^{\frac{-W_{i}(x)}{T}} \partial_{x}
$$

The operator $\mathcal{L}$ is hermitian since the matrix (A14) is symmetric and $\mathcal{L}_{i}$ itself is hermitian. The latter is easily verified by partial integration:

$$
\int_{0}^{l} d x q(x)\left(\partial_{x} e^{\frac{-W_{1}(x)}{T}} \partial_{x} p(x)\right)=\int_{0}^{l} d x p(x)\left(\partial_{x} e^{\frac{-W_{1}(x)}{T}} \partial_{x} q(x)\right) .
$$

\section{Onsager coefficients for many rigidly coupled motors}

Onsager coefficients can be calculated explicitly for a model of rigidly coupled motors. In this model, the two states are defined in the same way as before, but the current term in Eq. (13) is replaced by a convective term since all particles move with the same velocity. The transport equations are given by [11,8]

$$
\begin{aligned}
& \partial_{t} P_{1}+v \partial_{x} P_{1}=-\omega_{1}(x) P_{1}+\omega_{2}(x) P_{2} \\
& \partial_{t} P_{2}+v \partial_{x} P_{2}=\omega_{1}(x) P_{1}-\omega_{2}(x) P_{2},
\end{aligned},
$$

the velocity $v$ is determined by the force balance condition

$$
v=\xi^{-1}\left[f_{\text {ext }}-\int_{0}^{l} d x\left(P_{1} \partial_{x} W_{1}+P_{2} \partial_{x} W_{2}\right)\right] .
$$

Steady state distributions $P_{1}$ and $P_{2}=1 / l-P_{1}$ are solutions to

$$
v \partial_{x} P_{1}=\left(\omega_{1}+\omega_{2}\right) P_{1}+\omega_{2} / l .
$$

Using a power expansion in the velocity, $P_{1}$ can be written to lowest order

$$
P_{1}(x)=P_{1}^{(0)}(x)+P_{1}^{(1)}(x) v+O\left(v^{2}\right) \quad,
$$

with $P_{1}^{(0)}=\omega_{2} /\left(\omega_{1}+\omega_{2}\right) l$, and

$$
P_{1}^{(1)}=-\frac{1}{\omega_{1}+\omega_{2}} \partial_{x} P_{1}^{(0)} .
$$

As in the last section we use a reaction scheme with $\gamma_{\sigma}=$ 0 in order to keep the expressions simple. Also, without loss of generality we consider the case where $\alpha_{1}$ depends on $\Delta \mu$ but $\alpha_{2}$ remains constant. For small $\Delta \mu / T \ll 1$, we express the transition rates of Eq. (22) as

$$
\omega_{1}=\omega_{2} e^{-\Delta W / T}(1+\bar{\alpha}),
$$

where $\bar{\alpha} \equiv \alpha_{2} \Delta \mu / \omega_{2} T$. The force-velocity relation for small $v$ is given by

$$
f_{\text {ext }}=f^{(0)}+\left(\xi^{-1}+f^{(1)}\right) v+O\left(v^{2}\right),
$$

with coefficients

$$
f^{(n)}=-\int_{0}^{l} d x P_{1}^{(n)} \partial \Delta W .
$$

which depend on $\Delta \mu$. We can now calculate the Onsager coefficients. The effective friction

$$
\left.\lambda_{11} \equiv \frac{\partial v}{\partial f_{\text {ext }}}\right|_{\Delta \mu=0},
$$

can be determined from Eq. A23):

$$
\lambda_{11}^{-1}=\xi^{-1}+\frac{1}{l T} \int_{0}^{l} d x \frac{e^{-\Delta W / T}\left(\partial_{x} \Delta W\right)^{2}}{\omega_{2}\left(1+e^{-\Delta W / T}\right)^{3}} .
$$

Similarly,

$$
\left.\lambda_{12} \equiv \frac{\partial v}{\partial \Delta \mu}\right|_{f_{\mathrm{ext}}=0}=-\left.\lambda_{11} \frac{\partial f_{\mathrm{ext}}}{\partial \Delta \mu}\right|_{v=0},
$$

leads to 
$\lambda_{12}=-\left.\lambda_{11} \frac{\partial f^{(0)}}{\partial \Delta \mu}\right|_{\Delta \mu=0}=-\frac{\lambda_{11}}{l T} \int_{0}^{l} d x \frac{\alpha_{2} e^{-\Delta W / T} \partial_{x} \Delta W}{\omega_{2}\left(1+e^{-\Delta W / T}\right)^{2}}$

The second cross-coefficient

$$
\left.\lambda_{21} \equiv \frac{\partial r}{\partial f_{\mathrm{ext}}}\right|_{\Delta \mu=0}=\left.\lambda_{11} \frac{\partial r}{\partial v}\right|_{\Delta \mu=0},
$$

is determined from the fuel consumption rate $r(v, \Delta \mu)$. Using Eqns. (23), A29) and A21, we obtain

$$
\begin{aligned}
\left.\frac{\partial r}{\partial v}\right|_{\Delta \mu=0} & \left.=\int_{0}^{l} d x\left(\alpha_{2}+\alpha_{1}\right) \partial_{v} P_{1}\right]\left.\right|_{\Delta \mu=0} \\
& =-\frac{1}{l T} \int_{0}^{l} d x \frac{\alpha_{2} \partial_{x}(\Delta W) e^{-\Delta W / T}}{\omega_{2}\left(1+e^{-\Delta W / T}\right)^{2}},
\end{aligned}
$$

and thus as required $\lambda_{12}=\lambda_{21}$. Finally,

$$
\begin{aligned}
\lambda_{22} & =\left.\frac{\partial r}{\partial \Delta \mu}\right|_{f_{\mathrm{ext}}=0}=\left.\lambda_{12} \frac{\partial r}{\partial v}\right|_{\Delta \mu=0}+\left.\frac{\partial r}{\partial \Delta \mu}\right|_{v=0} \\
& =\frac{\lambda_{12}^{2}}{\lambda_{11}}+\left.\frac{\partial r}{\partial \Delta \mu}\right|_{v=0}
\end{aligned}
$$

which requires to calculate

$\left.\frac{\partial r}{\partial \Delta \mu}\right|_{v=0}=\int_{0}^{l}\left[\frac{\partial P_{1}^{(0)}}{\partial \Delta \mu}\left(\alpha_{1}+\alpha_{2}\right)+P_{1}^{(0)} \frac{\partial}{\partial \Delta \mu}\left(\alpha_{1}+\alpha_{2}\right)\right]$.

Using Eqns (22) and (23), we obtain

$$
\left.\frac{\partial r}{\partial \Delta \mu}\right|_{v=0}=\frac{1}{l T} \int_{0}^{l} d x\left[\frac{\alpha_{2} e^{-\Delta W / T}}{\left(1+e^{-\Delta W / T}\right)}\left(1-\frac{\alpha_{2}}{\omega_{2}}\right)\right] .
$$

Note that both $\lambda_{11}$ and $\lambda_{22}$ are positive while $\lambda_{12}$ can have either sign.

\section{APPENDIX B: DIFFUSION CLOSE TO A POTENTIAL MAXIMUM}

In Section IVD, we introduced the probabilities $p_{\sigma}(x)$ that a particle which initially starts at position $x$ close to a potential maximum will finally escape in the positive direction. We will calculate this probability for a piecewise linear potential as shown in Fig. 10 in the limit where the potential slopes extend to infinity which corresponds to large potential amplitudes $U / T \gg 1$. We consider the Fokker-Planck Equation

$$
\partial_{t} P+\partial_{x} J=0,
$$

with

$$
J=-\xi^{-1}\left[T \partial_{x} P+P \partial_{x} W-P f_{\mathrm{ext}}\right],
$$

for initial condition

$$
P(x, t=0)=\delta\left(x-x_{0}\right) .
$$

In order to determine what fraction of particles move to the right after a long time, we define the Laplace transform

$$
\tilde{P}(x, s)=\int_{0}^{\infty} d t P(x, t) e^{-s t}
$$

of the distribution and $\tilde{J}(x, s)=-\xi^{-1}\left[T \partial_{x} \tilde{P}+\tilde{P} \partial_{x} W-\right.$ $\left.\tilde{P} f_{\text {ext }}\right]$ of the current.

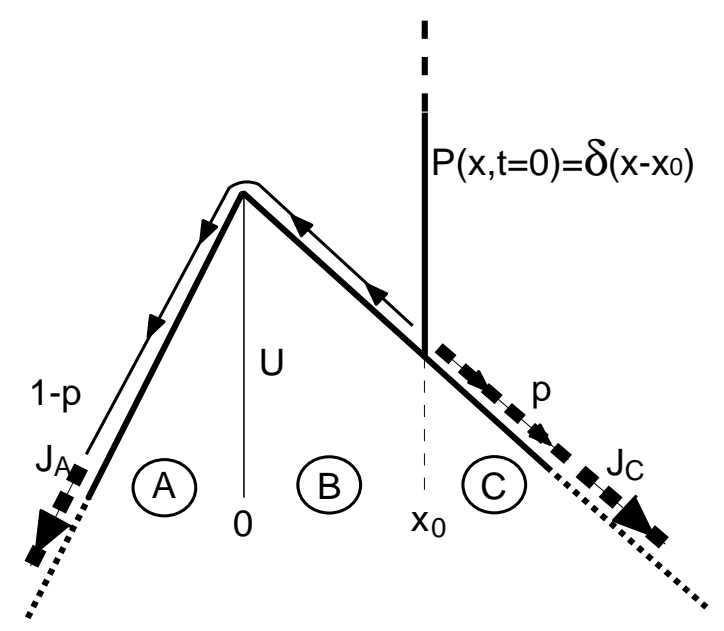

Figure 10. Schematic representation of diffusion near a potential maximum which can be divided in three different regions $\mathrm{A}, \mathrm{B}$ and $\mathrm{C}$. For a particle which initially appears at $x=x_{0}$, we are interested in the probability $p$ that it will finally move forward. This probability is related to the current $J_{C}$ which can be calculated in the limit of long times.

The average number of particles which pass at position $x$ after long times is given by

$$
\int_{0}^{\infty} d t J(x, t)=\tilde{J}(x, s=0)
$$

Noting that

$$
\int_{0}^{\infty} d t e^{-s t} \frac{\partial}{\partial t} P(x, t)=-P(x, 0)+s \tilde{P}(x, s),
$$

we obtain an equation for $\tilde{P}$ :

$$
\xi^{-1} \partial_{x}\left[T \partial_{x} \tilde{P}+\left(\partial_{x} W-f_{\text {ext }}\right) \tilde{P}\right]-s \tilde{P}=-P(x, 0)
$$

Since we are interested in $s=0$ we have to solve

$$
\partial_{x}\left[T \partial_{x} \tilde{P}+\left(\partial_{x} W-f_{\text {ext }}\right) \tilde{P}\right]=-\xi \delta\left(x-x_{0}\right) .
$$


All quantities of interest can be easily calculated if we we assume a piecewise linear potential

$$
W(x)=\left\{\begin{array}{ll}
U+f_{-} x & x<0 \\
U-f_{+} x & x \geq 0
\end{array},\right.
$$

with the potential slopes $f_{-}=U / a$ and $f_{+}=U /(l-a)$. We distinguish three different regions $\mathrm{A}, \mathrm{B}$ and $\mathrm{C}$ along the $x$-axis, see Fig. 10. Within each region, the solution to Eq. (B8) is

$$
\tilde{P}(x)=C_{0}+C_{1} e^{-\left(W(x)-x f_{\text {ext }}\right) / T},
$$

with two constants $C_{0}$ and $C_{1}$ which have to be determined for each of the three regions. We denote the corresponding solutions $\tilde{P}_{A}, \tilde{P}_{B}$ and $\tilde{P}_{C}$. Since we are looking for solutions which do not diverge for $x \rightarrow \pm \infty$, we have $C_{1}^{A}=C_{1}^{C}=0$ in regions $\mathrm{A}$ and $\mathrm{C}$ and therefore $\tilde{P}_{A}=C_{0}^{A}$ and $\tilde{P}_{C}=C_{0}^{C}$. This boundary condition for large $x$ can also be derived more carefully by first imposing the condition

$$
\lim _{x \rightarrow \pm \infty} \tilde{P}(x, s)=0
$$

for $s>0$ and taking the limit $s \rightarrow 0$ afterwards. Additional boundary conditions are the conditions of continuity of $\tilde{P}(x, 0)$ at $x=0$ and $x=x_{0}$

$$
\begin{aligned}
\tilde{P}_{A}(0) & =\tilde{P}_{B}(0) \\
\tilde{P}_{B}\left(x_{0}\right) & =\tilde{P}_{C}\left(x_{0}\right),
\end{aligned}
$$

and the matching conditions

$$
\begin{aligned}
\partial_{x} \tilde{P}_{A}(0) & =\partial_{x} \tilde{P}_{B}(0)-\tilde{P}_{A}(0)\left(f_{+}+f_{-}\right) / T \\
\partial_{x} \tilde{P}_{B}\left(x_{0}\right) & =\partial_{x} \tilde{P}_{C}\left(x_{0}\right)+\xi / T
\end{aligned}
$$

which follow from integrating Eq. (B8) at the singularities of the potential slope at $x=0$ and the delta-function at $x=x_{0}$. With these conditions, all free parameters can be determined and we obtain

$$
\begin{aligned}
C_{0}^{A} & =\frac{\xi}{f_{-}+f_{+}} e^{-\left(f_{+}+f_{\mathrm{ext}}\right) x_{0} / T} \\
C_{0}^{C} & =\frac{\xi}{f_{+}+f_{\mathrm{ext}}}\left[1-\frac{f_{-}-f_{\mathrm{ext}}}{f_{-}+f_{+}} e^{-\left(f_{+}+f_{\mathrm{ext}}\right) x_{0} / T}\right],
\end{aligned}
$$

The corresponding currents $\tilde{J}(x, s=0)$ are constant in region $\mathrm{A}$ and $\mathrm{C}$ :

$$
\begin{aligned}
& \tilde{J}_{A}=-\xi^{-1}\left(f_{-}-f_{\text {ext }}\right) C_{0}^{A} \\
& \tilde{J}_{C}=\xi^{-1}\left(f_{+}+f_{\text {ext }}\right) C_{0}^{C},
\end{aligned}
$$

which satisfy the normalization condition $J_{C}-J_{A}=1$. The probability $p\left(x_{0}\right)$ for forward motion of a particle which initially was at $x_{0}$ is equal to $J_{C}$ :

$$
p\left(x_{0}\right)=1-\frac{f_{-}-f_{\text {ext }}}{f_{-}+f_{+}} e^{-\left(f_{+}+f_{\text {ext }}\right) x_{0} / T} .
$$

The probabilities introduced in Eq. (45) are given by $p_{2}(0)=p(l-\delta), p_{1}(\delta)=p(\delta-a)$.
[1] B. Alberts, D. Bray, J. Lewis, M. Raff, K. Roberts, and J.D. Watson, 1994, The Molecular Biology of the Cell, (Garland, New York).

[2] A. Ajdari and J. Prost, C.R. Acad. Sci. Paris II 315, 1635 (1992).

[3] A. Ajdari, Ph.D. thesis (Université de Paris 6) (1992).

[4] M. O. Magnasco, Phys. Rev. Lett. 71, 1477 (1993).

[5] R.D. Astumian and M. Bier, Phys. Rev. Lett. 72, 1766 (1994).

[6] J. Prost, J.F. Chauwin, L. Peliti, and A. Ajdari, Phys. Rev. Lett. 72, 2652 (1994).

[7] R.D. Astumian, Science 276, 917 (1997).

[8] F. Jülicher, A. Ajdari, and J. Prost, Rev. Mod. Phys. 69 , 1269 (1997).

[9] R.P. Feynman, R.B. Leighton, and M. Sands, 1966, The Feynman lectures on physics (Addison-Wesley, Reading MA), Vol I, chap. 46.

[10] M.O. Magnasco, Phys. Rev. Lett. 72, 2656 (1994).

[11] F. Jülicher and J. Prost, Phys. Rev. Lett. 75, 2618 (1995).

[12] K. Sekimoto, J. Phys. Soc. Jap. 66, 1234 (1997).

[13] T. Shibata and S. Sasa, cond-mat/9710328.

[14] I. M. Sokolov and A. Blumen, J. Phys. A 30, 3021 (1997).

[15] F. Jülicher and R. Bruinsma, Biophys. J. 74, 1169 (1998).

[16] M. Matsuo and S. Sasa, cond-mat/9810220 (preprint).

[17] I. Derényi and D. Astumian, preprint (1998).

[18] J.M.R. Parrondo, J.M. Blanco, F.J. Cao, and R. Brito, cond-mat/9803302.

[19] T. Hondou, J. Phys. Soc. Jap. 67, 1818 (1998).

[20] H. Kamegawa, T. Hondou, and F. Takagi, Phys. Rev. Lett. 80, 5251 (1998).

[21] K. Sekimoto, Progress of theoretical Physics, Supplement No.130, 17 (1998).

[22] The quantities $v$ and $r$ are either ensemble averages or time averages. Experiments on single molecules and finite observation time require a special analysis (see for example [21]).

[23] T.L. Hill, Prog. Biophys. Mol. Biol. 28, 267 (1974).

[24] O. Kedem, and S.R. Caplan, Trans. Faraday. Soc. 61, 1897 (1965).

[25] M. Dogterom and B. Yurke, Science 278, 856 (1997).

[26] We assume that $\Delta \mu$ is changed by varying the ATP concentration while keeping $\mu_{A D P}$ and $\mu_{P}$ fixed.

[27] P.G. Bergmann and J. L. Lebowitz, Phys. Rev. 99, 578 (1955).

[28] S.R. De Groot, P. Mazur, 1962, Non-equilibrium thermodynamics (North Holland).

[29] K. Svoboda, C.F. Schmidt, B.J. Schnapp, and S.M. Block, Nature 365, 721 (1993).

[30] R.D. Vale, T. Funatsu, D.W. Pierce, L. Romberg, Y. Harada and T. Yanagida, Nature 380, 451 (1996).

[31] M. Schnitzer and S.M. Block, Nature 388, 386 (1997).

[32] W. Hua, E.C. Young, M.L. Fleming, and J. Gelles, Nature 388, 390 (1997).

[33] E. Mandelkow and A. Hoenger, Current opinion in Cell Biology 11, 34 (1999).

[34] S.M. Block, J. Cell. Biol. 140, 1281 (1998).

[35] W.O. Hancock and J. Howard, J. Cell Biol. 140, 1395 (1998).

[36] Y. Okada and N. Hirokawa, Science 283, 1152 (1999). 
[37] J.A. Spudich, Nature 348, 284 (1990).

[38] J. Howard, Nature 389, 561 (1997).

[39] M.J. Kushmerick, R. E. Larson, and R. E. Davies, Proc. R. Soc. London ser. B 175, 293 (1969).

[40] E. Eisenberg, T. Hill, and Y. Chen, Biophys. J. 29 , 195 (1980). 